Transplantation. 2013 Jan 27;95(2):372-7. doi: 10.1097/TP.0b013e31827220e7.

\title{
The impact of exercise training on liver transplanted familial amyloidotic polyneuropathy (FAP) patients.
}

Tomás MT, Santa-Clara H, Bruno PM, Monteiro E, Carrolo M, Barroso E, Sardinha LB, Fernhall

B.

Exercise and Health Laboratory, Interdisciplinary Centre for the Study of Human Performance, Faculty of Human Kinetics, Technical University of Lisbon, Cruz-Quebrada, Portugal. teresa.tomas@estesl.ipl.pt

Abstract

BACKGROUND: Liver transplantation is nowadays the only effective answer to adjourn the outcome of functional limitations associated with familial amyloidotic polyneuropathy (FAP), a neurodegenerative disease characterized by sensory and motor polyneuropathies. Nevertheless, there is a detrimental impact associated with the after-surgery period on the fragile physical condition of these patients. Exercise training has been proven to be effective on reconditioning patients after transplantation. However, the effects of exercise training in liver transplanted FAP patients have not been scrutinized yet.

METHODS: The study aimed to evaluate the effects of a 24-week exercise training program (supervised or home-based) on body composition, muscle strength, and walking capacity of liver transplanted FAP patients. To fulfill this goal, a sample corresponding to $33 \%$ of all FAP patients who undergone a liver transplantation in the area of Lisbon between January 2006 and December 2008 were followed over time. Three evaluation periods were accomplished: M1 (pre-exercise training period), M2 (immediate post-exercise training period), and M3 (24 weeks after M2). The former allowed an assessment of the impact of detraining in these patients.

RESULTS: The exercise training program improved body composition (lean mass and total body skeletal muscle mass), weight, and walking capacity. The improvements were more pronounced within the patients with supervised exercise training compared with the patients on the home-based program. In general, the benefits of the exercise training perdure even after a 24-week detraining period.

CONCLUSIONS: Exercise training results in significant improvements on the physical condition of liver transplanted FAP patients.

PMID: 23211558 [PubMed - indexed for MEDLINE] 